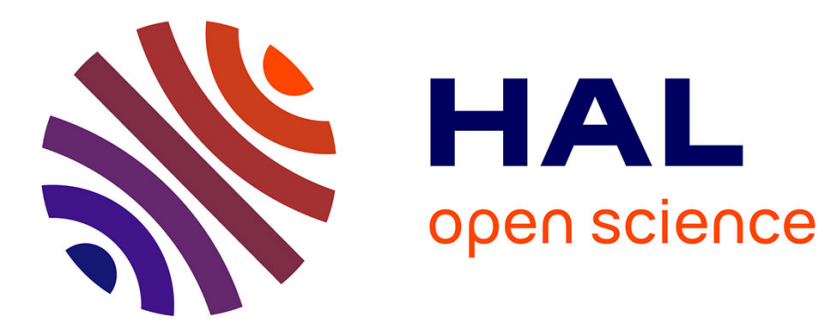

\title{
n-Refined Neutrosophic Groups I
}

Mohammad Abobala

\section{To cite this version:}

Mohammad Abobala. n-Refined Neutrosophic Groups I. International Journal of Neutrosophic Science, 2019, pp. 27 - 34. 10.5281/zenodo.3908405 . hal-02915734

\section{HAL Id: hal-02915734 \\ https://hal.science/hal-02915734}

Submitted on 20 Aug 2020

HAL is a multi-disciplinary open access archive for the deposit and dissemination of scientific research documents, whether they are published or not. The documents may come from teaching and research institutions in France or abroad, or from public or private research centers.
L'archive ouverte pluridisciplinaire HAL, est destinée au dépôt et à la diffusion de documents scientifiques de niveau recherche, publiés ou non, émanant des établissements d'enseignement et de recherche français ou étrangers, des laboratoires publics ou privés. 


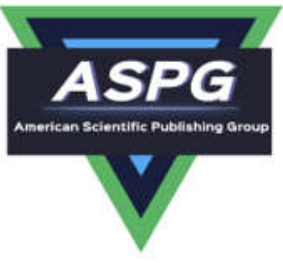

\title{
n-Refined Neutrosophic Groups I
}

\author{
Mohammad Abobala, Faculty of Science, Tishreen University, Lattakia, Syria \\ e-mail: mohammadabobala777@gmail.com
}

\begin{abstract}
The aim of this paper is to define for the first time the concept of n-refined neutrosophic group. This work is devoted to study some elementary properties of n-refined neutrosophic groups and to establish the algebraic basis of this structure such as n-refined neutrosophic subgroups, n-refined neutrosophic homomorphisms, and n-refined neutrosophic isomorphisms.
\end{abstract}

Keywords: n-Refined neutrosophic group, n-refined neutrosophic subgroup, n-refined neutrosophic homomorphism.

\section{Introduction}

Smarandache [2013] extended the neutrosophic set to refined [n-valued] neutrosophic set, and to refined neutrosophic logic, and to refined neutrosophic probability, i.e. the truth value $\mathrm{T}$ is refined/split into types of subtruths such as T1, T2, ., similarly indeterminacy I is refined/split into types of sub-indeterminacies I1, I2, .., and the falsehood F is refined/split into sub-falsehood F1, F2, ... . [9]. Neutrosophy as a new trend in logical studies founded by F. Smarandache provided interesting methods to deal with classical algebraic structures. Many neutrosophical algebraic structures came to light such as neutrosophic groups, neutrosophic rings, and neutrosophic loops. See $[1,2,4]$. Many studies were applied using the idea of splitting (refining) the indeterminacy I into two components $I_{1}, I_{2}$ with a multiplication operation defined by the following: $I_{1} \cdot I_{1}=I_{1}, I_{2} \cdot I_{2}=I_{2}, I_{1} \cdot I_{2}=I_{1}$, like refined neutrosophic groups. See [3]. This idea was used in soft computing in [8].

In $[5,6]$, Smarandache came with an interesting idea about splitting the indeterminacy $I$ into n-components $I_{1}, I_{2}, \ldots, I_{n}$, which refers to many different degrees of indeterminacy. In [7], Smarandache defined algebraic operations between refined neutrosophic numbers and refined neutrosophic sets. This refining idea will be very useful for us, since it helps with generalizing the concept of neutrosophic group.

In this work, we define the concept of n-refined neutrosophic group for the first time using formal multiplication between sub-indeterminacies. This multiplication is defined as follows:

$I_{i} \cdot I_{j}=I_{\min (i, j)}$. For example $I_{2} \cdot I_{5}=I_{2}$.

Also, concepts such as n-refined neutrosophic subgroups, homomorphisms, and AH-subgroups will be introduced.

\section{Preliminaries}

In the following section, we recall some important and useful definitions about neutrosophic groups.

DOI: $10.5281 /$ zenodo.3908405 


\section{Definition 2.1: $[1,5]$}

Let $\left(\mathrm{G},{ }^{*}\right)$ be a group . Then the neutrosophic group is generated by $\mathrm{G}$ and $\mathrm{I}$ under * denoted by $\mathrm{N}(\mathrm{G})=\{\langle G \cup I\rangle, *\}$.

$I$ is called the indeterminate (neutrosophic element) with the property $I^{2}=I$.

\section{Definition 2.2: [1]}

Let $\mathrm{N}(\mathrm{G})$ be a neutrosophic group and $\mathrm{H}$ be a neutrosophic subgroup, i.e ( $\mathrm{H}$ contains a proper subgroup of $\mathrm{G}$ ) of $\mathrm{N}(\mathrm{G})$. Then $\mathrm{H}$ is a neutrosophic normal subgroup of $\mathrm{N}(\mathrm{G})$ if $x H=H x$ for all $x \in N(G)$.

\section{Definition 2.3: [1]}

Let $N(G)$ be a neutrosophic group. Then the center of $N(G)$ is denoted by $C(N(G))$, and defined $C(N(G))=\{x \in N(G) ; x y=y x \forall y \in N(G)\}$

\section{Definition 2.4: [1]}

Let $\mathrm{N}(\mathrm{G}), \mathrm{N}(\mathrm{H})$ be two neutrosophic groups, then $N(G) \times N(H)=\{(g, h) ; g \in N(G), h \in N(H)\}$.

\section{Definition $2.5:[5]$}

Let $\mathrm{N}(\mathrm{G}), \mathrm{N}(\mathrm{H})$ be two neutrosophic groups and $\varphi: N(G) \rightarrow N(H)$ is called a neutrosophic homomorphism if it is a homomrphism between $\mathrm{G}, \mathrm{H}$ and $\varphi(I)=I^{\prime}$.

Where $I^{\prime}$ is the neutrosophic element of $\mathrm{N}(\mathrm{H})$.

If $\varphi$ is a correspondence one-to-one it is called a neutrosophic isomorphism.

\section{Main results}

In this section, we dicsuss our concepts and construct some related examples to clarify the validity of them.

\section{Definition 3.1:}

Let $(G, *)$ be a group, we define the corresponding n-refined neutrosophic group $N_{n}(G)$ as follows:

$N_{n}(G)=\left(<G \cup\left\{I_{1}, \ldots, I_{n}\right\}>, *\right)=\left\{\left(a_{0}, a_{1} I_{1}, \ldots, a_{n} I_{n}\right) ; a_{i} \in G\right\}$.

It is easy to see that $N_{n}(G)$ is closed under $*$, and it is a semi group but not a group since $I_{i}$ has no inverse with respect to $*$ in general.

\section{Remark 3.2:}

If $(\mathrm{G},+)$ is an additive abelian group, then addition on $N_{n}(G)$ can be described as follows:

Consider $x=\left(a_{0}, a_{1} I_{1}, \ldots, a_{n} I_{n}\right), y=\left(b_{0}, b_{1} I_{1}, \ldots, b_{n} I_{n}\right)$, we have $x+y=\left(a_{0}+b_{0},\left[a_{1}+b_{1}\right] I_{1}, \ldots,\left[a_{n}+b_{n}\right] I_{n}\right)$. In this case $\left(N_{n}(G),+\right)$ is a classical abelian group. 
The identity element is $(0,0, \ldots, 0)$.

It is easy to see that $N_{n}(G) \cong G \times G \times . . \times G(n+1$ times $)$ in the case of abelian additive group G.

\section{Example 3.3:}

Let $G=Z_{2}$ be the additive group of integers modulo 2, the corresponding 2-refined neutrosophic group is $N_{2}(G)=$ $\left\{(0,0,0),(1,0,0),\left(0, I_{1}, 0\right),\left(0,0, I_{2}\right),\left(1, I_{1}, 0\right),\left(1,0, I_{2}\right),\left(0, I_{1}, I_{2}\right),\left(1, I_{1}, I_{2}\right)\right\}$.

We clarify addition as follows:

\section{Remark 3.4:}

If $\mathrm{G}$ is a multiplicative group, then group product on $N_{n}(G)$ can be described as follows:

Consider $x=\left(a_{0}, a_{1} I_{1}, \ldots, a_{n} I_{n}\right), y=\left(b_{0}, b_{1} I_{1}, \ldots, b_{n} I_{n}\right)$, we have

$x y=\left(t_{0}, t_{1}, \ldots, t_{n}\right) ; t_{s}=\prod_{i, j=0}^{n}\left(a_{i} b_{j}\right) I_{i} I_{j} ; I_{0}=e_{G}$ and $I_{i} I_{j}=I_{s}$.

The identity element is $\left(e_{G}, e_{G} I_{1}, \ldots, e_{G} I_{n}\right)$.

In this case $N_{n}(G)$ is not isomorphic to the direct product of $\mathrm{n}+1$ copies of $\mathrm{G}$, since it is not a classical group in this case.

\section{Example 3.5:}

Let $G=S_{3}=\left\{f_{0}=I, f_{1}, f_{2}, f_{3}, f_{4}, f_{5}\right\}$ be the non abelian symmetric group of order 6 , the corresponding 2-refined neutrosophic group is $N_{2}(G)=\left\{\left(a, b I_{1}, c I_{2}\right) ; a, b, c \in G\right\}$.

We clarify the product on $N_{2}(G)$ as follows:

Consider $x=\left(f_{1}, f_{2} I_{1}, f_{0} I_{2}\right), y=\left(f_{2}, f_{1} I_{1}, f_{4} I_{2}\right)$, we have

$x y=\left(f_{1} f_{2},\left[\left(f_{1} f_{1}\right)\left(f_{2} f_{2}\right)\left(f_{2} f_{1}\right)\left(f_{2} f_{4}\right)\left(f_{0} f_{1}\right)\right] I_{1},\left[\left(f_{1} f_{4}\right)\left(f_{0} f_{2}\right)\left(f_{0} f_{4}\right)\right] I_{2}\right)$.

The identity is $\left(f_{0}, f_{0} I_{1}, f_{0} I_{2}\right)$.

\section{Definition 3.6:}

(a) Let $N_{n}(G)$ be an n-refined neutrosophic group. It is called abelian if $x * y=y * x$ for all $x, y \in N_{n}(G)$.

(b) The subset $Z\left(N_{n}(G)\right)=\left\{y \in N_{n}(G) ; y * x=x * y\right.$ for all $\left.x \in N_{n}(G)\right\}$ is called n-refined neutrosophic center.

\section{Theorem 3.7:}

Let $N_{n}(G)$ be an n-refined neutrosophic group. Then

(a) If $\mathrm{G}$ is abelian, $N_{n}(G)$ is abelian.

(b) $N_{n}(G)$ is abelian if and only if $N_{n}(G)=Z\left(N_{n}(G)\right)$.

Proof:

The proof is similar to the classical case. 


\section{Definition 3.8:}

Let $N_{n}(G)$ be an n-refined neutrosophic group, $\mathrm{H}$ be a nonempty subset of $N_{n}(G)$, we say that $\mathrm{H}$ is an n-refined neutrosophic subgroup if $\mathrm{H}$ contains a subgroup of $\mathrm{G}$.

\section{Example 3.9:}

Let $G=Z_{2}$ be the additive group of integers modulo 2, the corresponding 3-refined neutrosophic group is $N_{3}(G)$. The set $H=\left\{(0,0,0),(1,0,0),\left(1, I_{1}, 0\right),\left(1,0, I_{2}\right)\right\}$ is an n-refined neutrosophic subgroup of $N_{3}(G)$, since it contains $G=\{(0,0,0),(1,0,0)\}$ which is isomorphic to a subgroup of $\mathrm{G}$. (We can consider it as a subgroup of $\mathrm{G})$.

By previous example, we can see that Lagrange's theorem is not true in general in the case of finite n-refined neutrosophic group.

\section{Definition 3.10:}

Let $N_{n}(G)$ be an n-refined neutrosophic group, we denote to the number of elements in $N_{n}(G)$ by

$\mathrm{O}\left(N_{n}(G)\right)$. If $N_{n}(G)$ is finite, then $O\left(N_{n}(G)\right)=m$, elsewhere $O\left(N_{n}(G)\right)=\infty$

$O\left(N_{n}(G)\right)$ is called the order of n-refined neutrosophic group $N_{n}(G)$.

\section{Theorem 3.11:}

Let $\mathrm{G}$ be a finite group, $N_{n}(G)$ be its corresponding n-refined neutrosophic group. Then if $O(G)=m$, we have $O\left(N_{n}(G)\right)=m^{n+1}$.

Proof:

Since $N_{n}(G)=\left(<G \cup\left\{I_{1}, \ldots, I_{n}\right\}>, *\right)=\left\{\left(a_{0}, a_{1} I_{1}, \ldots, a_{n} I_{n}\right) ; a_{i} \in G\right\}$, we find that

$O\left(N_{n}(G)\right)=O(G) \times O(G) \times \ldots \times O(G)(n+1$ times $)=m^{n+1}$.

\section{Definition 3.12:}

Let $N_{n}(G), N_{n}(K)$ be two n-refined neutrosophic groups, $f: N_{n}(G) \rightarrow N_{n}(K)$ be a well defined map, we say that $f$ is an n-refined neutrosophic homomorphism if:

(a) $f(x y)=f(x) f(y)$ for all $x, y \in N_{n}(G)$.

(b) $f\left(e_{G}, e_{G}, \ldots, I_{k}, e_{G}, \ldots, e_{G}\right)=\left(e_{G}, e_{G}, \ldots, I_{k}, e_{G}, \ldots, e_{G}\right)$.

\section{Example 3.13:}

Let $G=Z, K=Z_{4}, f: N_{2}(G) \rightarrow N_{2}(K) ; f\left(x, y I_{1}, z I_{2}\right)=\left((x \bmod 4),(y \bmod 4) I_{1},(z \bmod 4) I_{2}\right)$

, where $x, y, z \in Z$.

Let $m=\left(x, y I_{1}, z I_{2}\right), n=\left(a, b I_{1}, c I_{2}\right)$ be two arbitrary elements in $N_{2}(G)$, it is clear that

$f(m+n)=f(m)+f(n)$. 
$f\left(I_{1}\right)=f\left(0,1 . I_{1}, 0 . I_{2}\right)=\left(0, I_{1}, 0\right), f\left(I_{2}\right)=f\left(0,0 . I_{1}, 1 . I_{2}\right)=\left(0,0, I_{2}\right)$. Thus $f$ is an n-refined neutrosophic homomorphism.

\section{Definition 3.14:}

Let $N_{n}(G), N_{n}(K)$ be two n-refined neutrosophic groups, $f: N_{n}(G) \rightarrow N_{n}(K)$ be an n-refined neutrosophic homomorphism, we define:

(a) $\operatorname{Ker}(f)=\left\{x \in N_{n}(G) ; f(x)=e_{N_{n}(K)}\right\}$.

(b) $\operatorname{Im}(f)=\left\{y \in N_{n}(K) ; \exists x \in N_{n}(G): f(x)=y\right\}$.

\section{Theorem 3.15:}

Let $N_{n}(G), N_{n}(K)$ be two n-refined neutrosophic groups, $f: N_{n}(G) \rightarrow N_{n}(K)$ be an n-refined neutrosophic homomorphism, we have:

(a) $\operatorname{Ker}(f)$ is a an n-refined neutrosophic subgroup of $N_{n}(G)$.

(b) $\operatorname{Im}(f)$ is an n-refined neutrosophic subgroup of $N_{n}(K)$.

Proof:

(a) Since the restriction $f_{G}$ of $f$ is a homomorphism between

$\mathrm{G}$ and $\mathrm{K}$, its kernel $\operatorname{Ker}\left(f_{G}\right)$ will be a subset of $\operatorname{Ker}(f)$, i.e $\operatorname{Ker}(f)$ contains a subgroup of $\mathrm{G}$, hence it is an nrefined neutrosophic subgroup according to Definition 3.8.

(b) The proof is similar to (a).

\section{Example 3.16:}

Let $G=Z, K=Z_{4}, f: N_{2}(G) \rightarrow N_{2}(K) ; f\left(x, y I_{1}, z I_{2}\right)=\left((x \bmod 4),(y \bmod 4) I_{1},(z \bmod 4) I_{2}\right)$

, where $x, y, z \in Z$.

$\operatorname{Ker}(f)=\left(4 Z, 4 Z I_{1}, 4 Z I_{2}\right)=\left\{4 x, 4 y I_{1}, 4 z I_{2} ; x, y, z \in Z\right\}$, which is a 2-refined neutrosophic subgroup, since it contains $L=4 Z$.

$\operatorname{Im}(f)=\left\{\left(a, b I_{1}, c I_{2}\right) ; a, b, c \in Z_{4}\right\}=N_{2}(K)$, which is a 2-refined subgroup, since it contains $S=Z_{4}$.

\section{Definition 3.17:}

Let $N_{n}(G), N_{n}(K)$ be two n-refined neutrosophic groups, $f: N_{n}(G) \rightarrow N_{n}(K)$ be an n-refined neutrosophic homomorphism, we call it an n-refined neutrosophic isomorphism if it is bijective.

\section{Example 3.18:}

Let $G=Z$ be the group of integers with normal addition, $N_{3}(G)=\left\{\left(a, b I_{1}, c I_{2}, d I_{3}\right) ; a, b, c, d \in Z\right\}$ be its corresponding 3-refined neutrosophic group. We define

$f: N_{3}(G) \rightarrow N_{3}(G) ; f\left(a, b I_{1}, c I_{2}, d I_{3}\right)=\left(-a, b I_{1}, c I_{2}, d I_{3}\right)$, it is clear that $f$ is a bijective n-refined neutrosophic homomorphism, thus it is an n-refined neutrosophic isomorphism. 


\section{Theorem 3.19:}

Let $N_{n}(G)$ be an n-refined neutrosophic group. The set $G I_{k}=\left\{x * I_{k} ; 1 \leq k \leq n\right\}$ has a group structure.

Proof:

We define the following operation on $G I_{k}$ :

$\left(x I_{k}\right) \times\left(y I_{k}\right)=(x * y) I_{k}$. It is east to check that $\times$ is well defined, associative with $I_{k}$ as an identity, and for each $x I_{k} \in G I_{k}$, there is an inverse $x^{-1} I_{k} ; x^{-1}$ is the inverse of $x$ in $\mathrm{G}$.

The previous group is called k-th pure neutrosophic component of $N_{n}(G)$.

\section{Example 3.20:}

Let $G=Z_{3}$ be the group of integers modulo 3 with respect to addition modulo 3,

$N_{3}(G)=\left\{\left(a, b I_{1}, c I_{2}, d I_{3}\right) ; a, b, c, d \in G\right\}$ be its corresponding 3-refined neutrosophic group.

The second pure neutrosophic subgroup of $N_{3}(G)$ is $G+I_{2}=\left\{I_{2}, 1+I_{2}, 2+I_{2}\right\}$.

The group's binary operation on $G+I_{2}$ is defined as follows:

$\left(x+I_{2}\right)+\left(y+I_{2}\right)=(x+y)+I_{2}$. For all $x, y \in G$.

\section{Theorem 3.21:}

Let $N_{n}(G)$ be an n-refined neutrosophic group, $G I_{k}$ be its k-th neutrosophic component. Then $G \cong G I_{k}$.

Proof:

Define $f: G I_{k} \rightarrow G ; f\left(x I_{k}\right)=x, f$ is a group isomorphism clearly, thus we get the proof.

\section{Definition 3.22:}

Let $G, H$ be two groups, $G \times H$ be the corresponding direct product. We define the direct product of the related nrefined neutrosophic groups as follows:

$N_{n}(G) \times N_{n}(H)=<G \times H \cup\left\{I_{1}, \ldots, I_{n}\right\}>$. We call it the n-refined neutrosophic direct product.

It is clear that $N_{n}(G) \times N_{n}(H)$ is an n-refined neutrosophic group, since $G \times H$ is a classical group.

\section{Example 3.23:}

Let $G=Z_{2}$ be the group of integers modulo 2 with respect to normal addition modulo 2, we construct the 2-refined neutrosophic direct product of $\mathrm{G}$ with itself.

$N_{2}(G) \times N_{2}(G)=<G \times G \cup\left\{I_{1}, I_{2}\right\}>=\left\{\left(a, b I_{1}, c I_{2}\right) ; a, b, c \in G \times G\right\}$.

We clarify addition on $N_{2}(G) \times N_{2}(G)$, consider $x=\left((1,0),(0,1) I_{1},(1,1) I_{2}\right), y=\left((0,0),(1,1) I_{1},(0,1) I_{2}\right)$.

We have: $x+y=\left((1,0),(1,0) I_{1},(1,0) I_{2}\right)$. 


\section{Definition 3.24:}

Let $N_{n}(G)=\left\{\left(a_{0}, a_{1} I_{1}, \ldots, a_{n} I_{n}\right) ; a_{i} \in G\right\}$ be an n-refined neutrosophic group,

$N_{n}(H)=\left\{\left(b_{0}, b_{1} I_{1}, \ldots, b_{n} I_{n}\right) ; b_{i} \in H_{i} ; H_{i}\right.$ is a subgroup of $G$ for all $\left.i\right\}$ is called an AH-subgroup of $N_{n}(G)$.

If $H_{i} \cong H_{j}$ for all $i \neq j$, then it is called an AHS-subgroup.

The AH-subgroup $N_{n}(H)$ is called AH-abelian if $H_{i}$ is abelian for all $i$. Also, it is called AH-cyclic if $H_{i}$ is cyclic for all $i$.

\section{Example 3.25:}

Let $G=S_{3}$ be the non abelian symmetric group of order 6, there are two non isomorphic subgroups of G,

$K \cong Z_{2}, S \cong Z_{3}$, consider the corresponding 3-refined neutrosophic group $N_{3}(G)$, we have:

$N_{3}(H)=\left(K, S I_{1}, K I_{2}, S I_{3}\right)=\left\{\left(a, b I_{1}, c I_{2}, d I_{3}\right) ; a, c \in K\right.$ and $\left.b, d \in S\right\}$ is an AH-subgroup of $N_{3}(G)$.

$N_{3}(H)$ is an AH-cyclic, since $K, S$ are cyclic.

\section{Conclusion}

In this article we have defined the concept of n-refined neutrosophic group for the first time. Also, we have introduced some corresponding notions such as n-refined neutrosophic subgroup, n-refined neutrosophic homomorphism, and n-refined neutrosophic isomorphism. Many examples were constructed to clarify these concepts.

\section{Future researches}

This work has established the theory of n-refined neutrosophic groups. In future works, we aim to define normality, quotients, and to study $\mathrm{AH}$-substructures in n-refined neutrosophic groups.

Funding: "This research received no external funding"

Conflicts of Interest: "The authors declare no conflict of interest."

\section{References}

[1] Agboola, A.A.A,. Akwu, A.D,. and Oyebo, Y.T,. "Neutrosophic Groups and Subgroups", International .J .Math.Combin, Vol. 3, pp. 1-9, 2012.

[2] Agboola, A.A.A., Akinola, A.D., and Oyebola, O.Y.," NeutrosophicRings I ", International J.Mathcombin, Vol. 4, pp. 1-14, 2011.

[3] Agboola, A.A.A., "On Refined Neutrosophic Algebraic Structures", Neutrosophic Sets and Systems, Vol. 10, pp. 99-102, 2015.

[4] Kandasamy, V.W.B., and Smarandache, F., "Some Neutrosophic Algebraic Structures and Neutrosophic NAlgebraic Structures", Hexis, Phonex, Arizona 2006.

[5] Smarandache, F., "Symbolic Neutrosophic Theory", EuropaNova asbl, Bruxelles, 2015. 
[6] Smarandache, F., "n-Valued Refined Neutrosophic Logic and Its Applications in Physics", Progress in Physics, Vol. 4, pp.143-146, 2013.

[7] Smarandache, F., "Refined Literal Indeterminacy and the Multiplication Law of Sub-Indeterminacies", Neutrosophic Sets ans Systems, Vol. 9, 2015.

[8] Deli, I., " Refined Neutrosophic Sets and Refined Neutrosophic Soft Sets: Theory and Applications", Hand Book of Research on generalized and Hybrid Set Structures and Applications for Soft Computing, 2016.

[9] Smarandache, Florentin, n-Valued Refined Neutrosophic Logic and Its Applications in Physics, Progress in Physics, 143-146, Vol. 4, 2013; https://arxiv.org/ftp/arxiv/papers/1407/1407.1041.pdf http://fs.unm.edu/n-ValuedNeutrosophicLogic-PiP.pdf 